\section{Mécanismes de la perception gustative des lipides alimentaires}

Naim Akhtar Khan, Dany Gaillard, Abdelghani દl-Yassimi, Patricia Passilly-Degrace, Aziz Hichami, Philippe Besnard
N.A. Khan, A. El-Yassimi, A. Hichami :

EA4183 Lipides et Signalisation Cellulaire ;

Université de Bourgogne, Dijon.

Faculté des Sciences de la Vie,

6, boulevard Gabriel, 21000 Dijon, France.

naim.khan@u-bourgogne.fr

D. Gaillard, P. Passilly-Degrace, P. Besnard:

Physiologie de la Nutrition, UMR Inserm U866,

École Nationale Supérieure

de Biologie appliquée à la Nutrition

et à l'Alimentation,

Université de Bourgogne, Dijon, France.
> L'obésité constitue indéniablement un des problèmes majeurs de santé publique de ce début du XXI ${ }^{e}$ siècle. Sa prévalence est en constante augmentation notamment chez les enfants. Ce constat n'est pas anodin car l'obésité est souvent associée à diverses pathologies graves (atteintes vasculaires, diabète de type 2 , hypertension, cancer). L'opulence alimentaire, en nous permettant de satisfaire nos appétits spécifiques, contribue à ce phénomène. C'est ainsi que dans le régime occidental, les lipides alimentaires représentent près de $40 \%$ des apports caloriques journaliers alors que les recommandations nutritionnelles sont inférieures de 5 à $10 \%$. L'origine de cette attirance pour les corps gras, encore mal connue, est également observée chez la souris. Dans cette espèce, on a longtemps pensé que la perception oro-sensorielle des lipides alimentaires dépendait uniquement de leurs propriétés olfactives et texturales. Cette vision quelque peu restrictive a récemment été battue en brèche par une série d'expériences montrant que la gustation joue également un rôle important dans la préférence spontanée pour les corps gras.

\section{CD36 récepteur \\ des acides gras à longue chaîne}

Le goût informe le système nerveux central sur la qualité des aliments ingérés conduisant ainsi à des comportements stéréotypés (attraction ou rejet) [1]. La perception des saveurs s'effectue par le biais de cellules réceptrices spécialisées (taste receptor cells, TRC) localisées dans les bourgeons du goût des papilles gustatives présentes essentiellement dans l'épithélium lingual. Nous avons récemment montré que la protéine CD36, connue pour lier les acides gras à longue chaîne (AGLC, nombre de carbones $\geq 16$ ) avec une très haute affinité, est abondamment exprimée au niveau de certaines TRC chez la souris [2, 9]. Cette

Figure 1. Mécanismes d'action des acides gras à longue chaîne (AGLC) dans l'activation des cellules gustatives CD36-positives de souris : modèle de travail. L'interaction AGLC/CD36 va induire la phosphorylation de Src-PTK particulièrement de Fyn- $p^{59}$ et Yes- $p^{62}$, qui, à leur tour, activent la phospholipase C, PLC (par un mécanisme encore inconnu) qui hydrolyse la phosphatidyl-inositol bisphosphate, libérant ainsi I'IP qui provoque la libération du $\mathrm{Ca}^{2+}$ libre à partir du réticulum endoplasmique. Ce phénomène est suivi par l'influx calcique via l'ouverture des canaux SOC. Les Src-PTK particulièrement Fyn- ${ }^{59}$ et Yes- $p^{62}$, régulent l'activation de ces canaux calciques. Une augmentation

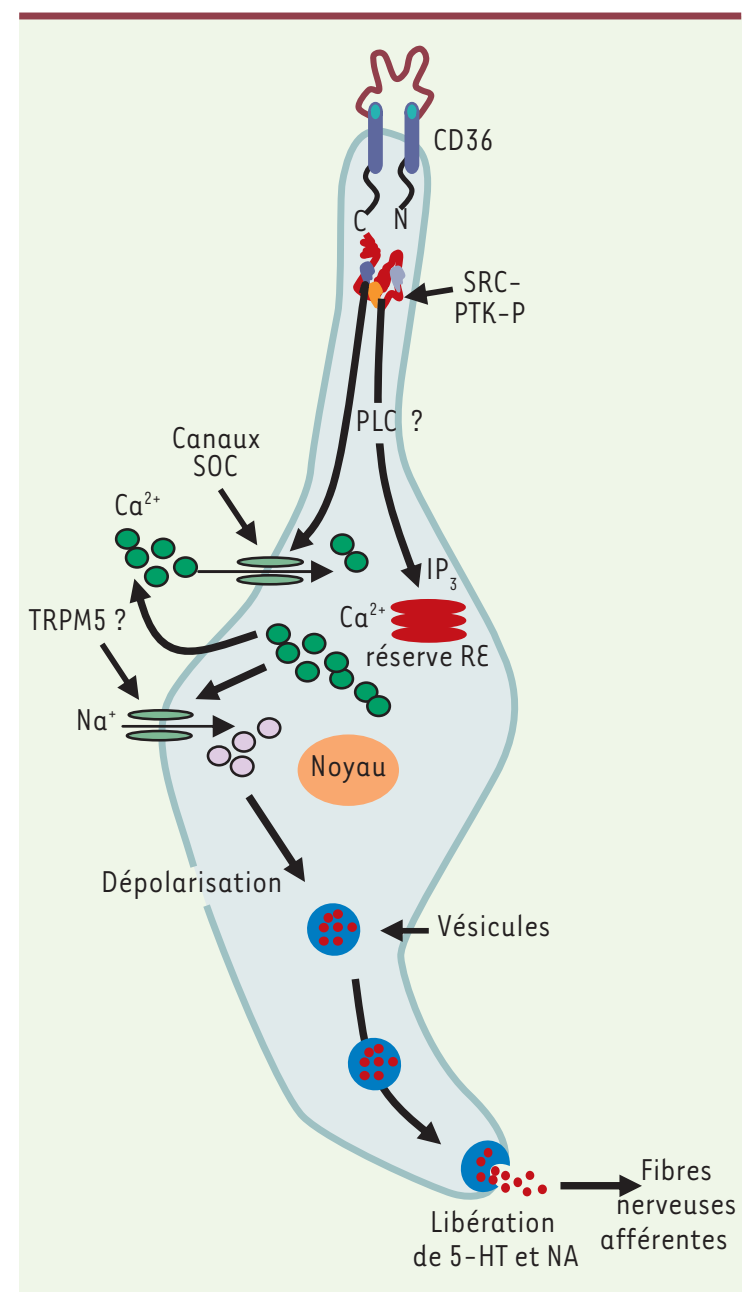
massive du calcium intracellulaire va entraîner l'exocytose de 5-HT et de NA qui transmettront le signal vers les fibres afférentes, en relayant l'information vers le cerveau sur la qualité et la quantité de la gustation lipidique lors d'une prise alimentaire. glycoprotéine transmembranaire s'est avéré être impliquée dans la perception des lipides. En effet, l'invalidation du gène codant pour le CD36 diminue de façon drastique la préférence spontanée 
pour les AGLC chez la souris, sans pour autant affecter sa perception du sucré ou de l'amer [2]. Bien que les graisses alimentaires soient très majoritairement composées de triglycérides, ce sont donc les AGLC qui sont responsables de l'attirance spontanée pour les lipides. Mais comment sont-ils libérés dans la cavité buccale? L'équipe de Kawai et Fushiki a répondu à cette question en démontrant qu'il existe chez la souris une lipase linguale extrêmement efficace, capable de libérer en quelques secondes un grand nombre d'AGLC à partir des triglycérides alimentaires [3].

\section{CD36, signalisation calcique} et libération de neurotransmetteurs dans les cellules réceptrices du goût Les mécanismes moléculaires à l'origine de la perception des différentes saveurs (sucré, salé, acide, amer et umami) ont fait l'objet de nombreuses études. Quelle que soit la molécule sapide, son interaction avec sa protéine réceptrice au niveau des TRC aboutit à une augmentation de la concentration du calcium libre intracellulaire $\left(\left[\mathrm{Ca}^{2+}\right] \mathrm{i}\right)$ à l'origine de la libération de neuromédiateurs au niveau des afférences nerveuses gustatives [1]. Qu'en est-il des AGLC? Pour tenter de répondre à cette question, nous avons entrepris l'isolement par sélection positive de cellules TRC exprimant CD36 (billes magnétisées couplées à un anticorps anti-CD36) à partir des papilles gustatives de souris [4]. Cette approche inédite nous a permis de montrer que les AGLC induisent également une augmentation du $\left[\mathrm{Ca}^{2+}\right] \mathrm{i}$ dans les TRC. Ce phénomène est spécifique au CD36 car la signalisation calcique est fortement déprimée en présence d'un inhibiteur pharmacologique de la liaison des AGLC sur le CD36 [4]. Nous avons commencé à décrypter la cascade de signalisation induite par les AGLC [5]. L'augmentation $\mathrm{du}\left[\mathrm{Ca}^{2+}\right] \mathrm{i}$ semble résulter d'une mobilisation AGLC-dépendante du calcium intracellulaire à partir du réticulum endoplasmique via la production d'inositol tri-phosphate $\left(I_{3}\right)$ et l'ouver- ture des canaux SOC (store-operated calcium) favorisant ainsi l'influx calcique [5]. Ce dernier phénomène semble être la conséquence de la phosphorylation de tyrosines kinases de la famille Src $\left(F y n-p^{59}\right.$ et Yes- $\left.p^{62}\right)$ en réponse à I'interaction AGLC/CD36 au niveau des TRC (Figure 1). Comme pour le sucré, l'amer ou l'umami [6], l'augmentation $\mathrm{du}\left[\mathrm{Ca}^{2+}\right] \mathrm{i}$ induite par les AGLC semble affecter le fonctionnement des canaux sodiques/potassiques TRPM5 (transient receptor potentiel melastatin-5) induisant ainsi une dépolarisation cellulaire à l'origine de la libération de neuromédiateurs (Figure 1). En effet, la préférence pour les $A G L C$ est perdue chez les souris TRPM5 $5^{-/-}$[7].

Nous avons également montré que les TRC CD36-positives expriment les gènes codant pour les enzymes clés de la synthèse des neurotransmetteurs monoaminergiques que sont la nor-adrénaline (NA) et la sérotonine (5-hydroxytryptamine, 5-HT) [5]. Ce système est fonctionnel, au moins in vitro, puisqu'en présence d'AGLC, les TRC CD36-positives libèrent de la 5-HT et de la NA (Figure 1). Cependant, il reste à démontrer que les cellules CD36-positives peuvent établir des contacts synaptiques avec les afférences gustatives. II est très vraisemblable que ce soit le cas puisque la section bilatérale des nerfs gustatifs (corde du tympan et glossopharyngien) s'accompagne d'une perte de l'attraction pour les sources lipidiques chez la souris [4]. De plus, un dépôt oral d'AGLC entraîne chez la souris une forte activation neuronale au niveau du noyau du tractus solitaire, premier relais de la cascade gustative au niveau du cerveau. Ce phénomène est strictement dépendant de la présence du CD36 car il n'est plus reproduit chez les animaux $C D 36^{-/-}[4]$.

\section{Conclusion}

Chez la souris, nos travaux démontrent donc que le CD36 lingual est un lipidorécepteur gustatif participant à la couverture des besoins énergétiques de l'organisme en sélectionnant les nutriments lipidiques. Cette fonction inédite pourrait constituer un avantage nutritionnel non négligeable en cas de restriction alimentaire chronique. $\varepsilon$ n effet, les aliments riches en lipides sont connus pour avoir une forte densité énergétique et être les vecteurs des acides gras indispensables et des vitamines liposolubles $(A, D, \varepsilon, K)$ dont les rôles biologiques sont essentiels et multiples. En revanche, elle pourrait participer à la mise en place d'une surcharge pondérale en cas de pléthore alimentaire permanente. On ignore actuellement si une fonction similaire existe chez l'Homme. Le fait que des sujets adultes en bonne santé soient capables de détecter de façon spécifique et avec un seuil de perception très bas la présence d'AGLC dans des repas fictifs (car non ingérés) étaye cette hypothèse [8]. L'identification de marqueurs biologiques pertinents et non invasifs devrait permettre de répondre à cette importante question dans un proche avenir. $\diamond$ Unraveling the downstream signalling of gustatory perception of lipids

\section{RÉFÉRENCES}

1. Chandrashekar J, Hoon MA, Ryba NJ, Zuker CS. The receptors and cells for mammalian taste. Nature 2006 ; 444 : 288-94.

2. Laugerette F, Passilly-Degrace P, Patris B, et al. CD36 involvement in orosensory detection of dietary lipids, spontaneous fat preference, and digestive secretions. J Clin Invest 2005 ; 115 : 3177-84.

3. Kawai T, Fushiki T. Importance of lipolysis in oral cavity for orosensory detection of fat. Am J Physiol Regul Integr Comp Physiol 2003 ; 285 : R447-54.

4. Gaillard D, Laugerette F, Darcel N, et al. The gustatory pathway is involved in CD36-mediated orosensory perception of long-chain fatty acids in the mouse. FASEB J 2008 ; 22 : 1458-68.

5. El-Yassimi A, Hichami A, Besnard P, Khan NA. Linoleic acid induces calcium signaling, Src-kinase phosphorylation $B$. and neurotransmitters release in mouse CD36-positive gustatory cells. J Biol Chem 2008 ; 283 : 12949-59.

6. Sugita M. Taste perception and coding in the periphery. Cell Mol Life Sci 2006 ; 63 : 2000-15.

7. Sclafani A, Zukerman S, Glendinning JL, Margolskee RF. Fat and carbohydrate preferences in mice: the contribution of alpha-gustducin and Trpm5 tastesignaling proteins. Am J Physiol Regul Integr Comp Physiol 2007 ; 293 : R1504-13.

8. Chale-Rush A, Burgess JR, Mattes RD Evidence for human orosensory (taste?) sensitivity to free fatty acids. Chem Senses 2007 ; 32 : 423-31.

9. Laugerette F, Passilly-Degrace P, Patris B, et al. CD36, un sérieux jalon sur la piste du goût du gras. Med Sci (Paris) $2006 ; 22: 357-9$. 